\title{
Low circulating arachidonic acid is associated with macroalbuminuria in diabetic patients: a cross-sectional examination of the KAMOGAWA-DM cohort study
}

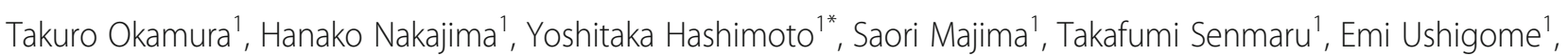
Naoko Nakanishi ${ }^{1}$, Masahide Hamaguchi ${ }^{1}$, Mai Asano ${ }^{1}$, Masahiro Yamazaki ${ }^{1}$, Hiroshi Takakuwa ${ }^{2}$ and Michiaki Fukui ${ }^{1}$

\begin{abstract}
Background: Diabetic nephropathy, a major complication of diabetes, is the primary risk factor for dialysis, cardiovascular diseases, and mortality. Dietary fatty acids (FAs) have been revealed to be related with cardiovascular diseases in the general populations. The aim of this study was to investigate the association of circulating FAs with diabetic nephropathy.

Methods: In this cross-sectional study, 190 Japanese patients with type 2 diabetes were included. Circulating FAs were measured by gas chromatography-mass spectrometry. Spearman rank correlation coefficients were used to investigate the association between the logarithm of FAs and the logarithm of urinary albumin excretion (UAE). We have performed logistic regression analysis to determine the effect of FAs on the presence of macroalbuminuria, defined as UAE value $\geq 300 \mathrm{mg} / \mathrm{g}$ creatinine.

Results: Mean age, body mass index, and duration of diabetes were $62.7 \pm 12.1$ years, $25.0 \pm 4.5 \mathrm{~kg} / \mathrm{m}^{2}$, and $9.8 \pm$ 8.7 years, respectively. In total, 26 patients were diagnosed with macroalbuminuria. The logarithm of circulating arachidonic acid (AA) was negatively associated with the logarithm of UAE $(r=-0.221, p=0.002)$. Additionally, circulating $A A$ in patients with macroalbuminuria was lower than that in patients without macroalbuminuria $(112.3 \pm 75.3 \mathrm{mg} /$ day vs. $164.8 \pm 66.0 \mathrm{mg} /$ day, $p<0.001)$. The logarithm of circulating AA was associated with the presence of macroalbuminuria after adjusting for covariates (odds ratio of $\Delta 1$ incremental: $0.32,95 \%$ confidence interval: 0.10-0.99, $p=0.042$ ).
\end{abstract}

Conclusions: Circulating AA was negatively associated with UAE and the presence of macroalbuminuria.

Keywords: Cohort study, Epidemiology, Fatty acids, Arachidonic acid, Diabetic nephropathy

\footnotetext{
* Correspondence: -hashi@koto.kpu-m.ac.jp

'Department of Endocrinology and Metabolism, Kyoto Prefectural University of Medicine, Kyoto 602-8566, Japan

Full list of author information is available at the end of the article
} 


\section{Background}

Diabetic nephropathy is a major complication of type 2 diabetes mellitus (T2DM), and is the most common cause of end-stage renal disease. The prevalence of diabetic nephropathy is increasing worldwide [1]. Further, diabetic nephropathy, especially macroalbuminuria, is reported to be the primary risk factor for cardiovascular disease [2], and, eventually, mortality [3]. Thus, the prevention and treatment of diabetic nephropathy, especially macroalbuminuria, are important for the prevention of renal and cardiovascular events and death.

The role of fatty acids (FAs) in inflammation and related chronic diseases is well established. Originally, albumin is an FA transport protein, and hyperfiltration in diabetes causes a high concentration of FAs to enter the tubular space, along with albumin [4]. Circulating FAs are composed of saturated FAs without double bonds, monounsaturated FAs with a single double bond, and polyunsaturated FAs (PUFAs) with multiple double bonds. Several studies have reported that increased influx of these FAs, especially saturated FAs, contributes to the worsening of tubular damage $[5,6]$. In addition to saturated FAs, we should focus on the effects of the other types of FAs in organ damage through lipotoxicity. Previous studies reported that n-3 PUFAs, such as eicosapentaenoic acid (EPA) and docosahexaenoic acid, have anti-inflammatory and cardiovascular protective properties [7-9]. On the other hand, n-6 PUFA, such as arachidonic acid (AA), produces a series of lipid mediators called eicosanoids (prostaglandins and leukotrienes), which play a central role in enhancing vascular permeability, and neutrophil infiltration and activation during the early stages of the inflammatory response [10]. In addition, a low ratio of serum EPA to AA has been reported to be a risk factor for cardiovascular events [11-13]. Conversely, several previous studies demonstrated that moderate intake of dietary n-6 PUFAs lowers the risk of coronary artery disease [14] and circulating n-6 PUFAs were inversely associated with total mortality and coronary artery disease mortality [15].

In recent years, it has become clear that the concentration of each circulating FAs in patients with diabetes differed from those of people without diabetes and that these differences may contribute to the development of organ damage [16-18]. However, the association between these circulating FAs, especially detail components of FAs, and diabetic nephropathy has not yet been clarified.

Thus, we conducted the present cross-sectional study with an aim to determine the relation between circulating FAs and diabetic nephropathy.

\section{Methods}

Study design and participants

The KAMOGAWA-DM cohort study, is an ongoing prospective cohort study that began in 2014 [19], included the outpatient clinics of the Kyoto Prefectural University of Medicine (Kyoto, Japan) and the Kameoka Municipal Hospital (Kameoka, Japan). For the present cross-sectional study, we included T2DM patients in whom FA measurements were performed. We excluded patients taking EPA medication. This study was approved by the hospital's Ethics Committee. T2DM was diagnosed based on the criteria by the American Diabetes Association [20].

\section{Data collection}

The background factors of patients (i.e., sex, age, disease duration, smoking, and the history of cardiovascular disease (CVD)) was gathered from their medical records. CVD was defined as myocardial infarction and stroke. Blood pressure was measured in an outpatient setting. After an overnight fast, venous blood samples were collected from the patients and fasting plasma glucose, triglycerides, total cholesterol, high-density lipoprotein cholesterol, low-density lipoprotein cholesterol, creatinine, uric acids, and C-peptide were measured. Hemoglobin A1c (HbA1c) levels were measured by high-performance liquid chromatography, ant the values are presented as the National Glycohemoglobin Standardization Program unit. An index of insulin resistance using serum C-peptide concentration, 20/(fasting C-peptide $\times$ fasting plasma glucose) [21], was used. All patients submitted spot morning urine samples, and urinary albumin excretion (UAE) was measured by immunoturbidimetric assay. Mean UAE in the present study was used as the average of the three urinary measurements. Macroalbuminuria was defined as UAE > $300 \mathrm{mg} / \mathrm{g}$ creatinine [22].

Diabetic neuropathy was diagnosed based on the criteria by The Diagnostic Neuropathy Study Group [23]. Retinopathy was classified into four categories according to the ophthalmologist's diagnosis as follows: NDR, no diabetic retinopathy; SDR, simple diabetic retinopathy; PPDR, pre-proliferative diabetic retinopathy; and PDR, proliferative diabetic retinopathy.

\section{Estimation and assessment of the patients' habitual diet and nutritional intake}

A brief-type self-administered diet history questionnaire (BDHQ) was adopted for the assessment of each patient's habitual diet and nutritional intake [24]. The details of the BDHQ are provided elsewhere [24]. In brief, the BDHQ estimates the dietary intake of 58 food items over the past month from respondents' memories using a computer algorithm, based on the Standard Tables of Food Composition in Japan [25]. And then, the dietary total energy ( $\mathrm{kcal} /$ day), carbohydrate (g/day), protein (g/ day), total fat (g/day), saturated FAs (g/day), monosaturated FAs (g/day), PUFAs (g/day), n-3 FAs (g/day), n-6 


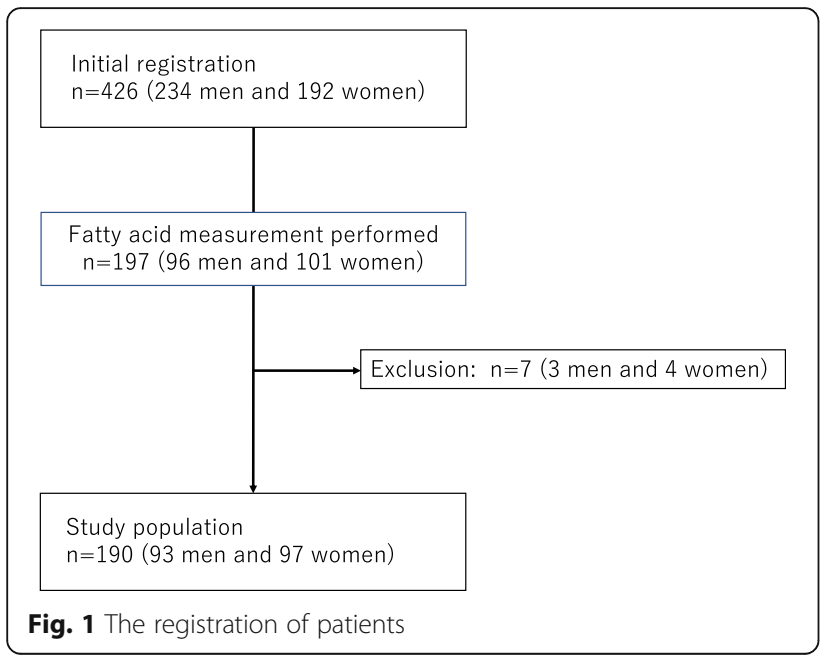

FAs (g/day), total fiber (g/day), cholesterol (g/day), alcohol (g/day), and sodium (g/day) intake were estimated using this calculation program of the BDHQ.

\section{Measurement of circulating free fatty acids}

The composition of FAs in frozen serum samples was measured by GC/MS, Agilent 7890B/5977B (Agilent Technologies, Santa Clara, CA, USA). We metylated $25 \mu \mathrm{l}$ of serum using a FA methylation kit (Nacalai Tesque, Kyoto, Japan), and loaded the final product onto a Varian capillary column (DB-FATWAX UI; Agilent Technologies). The capillary column used for FA separation was CP-Sil 88 for FAME $(100 \mathrm{~m} \times$ an inner diameter of $0.25 \mathrm{~mm} \times$ membrane thickness of $0.20 \mu \mathrm{m}$, Agilent Technologies). We set the temperature in column at $100^{\circ} \mathrm{C}$ for $4 \mathrm{~min}$ and then increased gradually by $3{ }^{\circ} \mathrm{C} / \mathrm{min}$ to $240{ }^{\circ} \mathrm{C}$ and held there for $7 \mathrm{~min}$. We injected the samples in split mode at a split ratio of 5:1. Each FA methyl ester was detected in the selected ion monitoring mode. All results were normalized to the peak height of the C17:0 internal standard [26].

\section{Statistical analysis}

JMP (ver. 13.0) software (SAS Institute, Cary, NC, USA) was used for all analyses of the patients' data. Probability values of $<0.05$ were accepted as significant. Categorical variables were expressed as numbers, and continuous variables were presented as the mean $\pm \mathrm{SD}$. To assess the statistical significance of the differences between groups, the chi-square test for categorical variables and the Wilcoxon signed-rank test for continuous variables was used because the continuous variables did not follow a normal distribution. Spearman rank correlation coefficients were used to investigate the association between the logarithm of FAs and the logarithm of UAE. Then, because AA was found to be significantly correlated with the logarithm UAE, the odds ratio (OR) and 95\%
Table 1 Clinical characteristics of the study patients

\begin{tabular}{|c|c|}
\hline & Total $(n=190)$ \\
\hline Sex, men/women & 93/97 \\
\hline Age, yrs & $65.0(8.0)$ \\
\hline Duration of diabetes, yrs & $7.0(11.0)$ \\
\hline $\mathrm{BMI}, \mathrm{kg} / \mathrm{m}^{2}$ & $24.4(5.1)$ \\
\hline Systolic blood pressure, $\mathrm{mmHg}$ & $133.0(22.0)$ \\
\hline Diastolic blood pressure, $\mathrm{mmHg}$ & $81.0(15.0)$ \\
\hline Fasting plasma glucose, $\mathrm{mmol} / \mathrm{L}$ & $6.9(2.2)$ \\
\hline Hemoglobin A1c, \% & $7.1(1.7)$ \\
\hline C-peptide, $\mathrm{nmol} / \mathrm{L}$ & $0.5(0.4)$ \\
\hline 20/C-peptide/fasting plasma glucose & $5.9(4.2)$ \\
\hline Triglycerides, $\mathrm{mmol} / \mathrm{L}$ & $1.3(0.6)$ \\
\hline Total cholesterol, mmol/L & $5.2(1.1)$ \\
\hline High-density lipoprotein cholesterol, mmol/L & $1.5(0.5)$ \\
\hline Low-density lipoprotein cholesterol, mmol/L & $3.0(0.9)$ \\
\hline Creatinine, $\mu \mathrm{mol} / \mathrm{L}$ & $66.7(26.6)$ \\
\hline Estimated GFR, mL/min/1.73m² & $71.7(22.6)$ \\
\hline Uric acids, mmol/L & $306.3(107.1)$ \\
\hline Urine albumin to creatinine ratio, $\mathrm{mg} / \mathrm{gCr}$ & $20.4(66.6)$ \\
\hline Diabetic nephropathy & $115 / 49 / 26$ \\
\hline Normoalbuminuria & $115(60.5)$ \\
\hline Microalbuminuria & $49(25.8)$ \\
\hline Macroalbuminuria & $26(13.7)$ \\
\hline Diabetic retinopathy, NDR/SDR/PPDR/PDR & $163 / 13 / 4 / 10$ \\
\hline Diabetic neuropathy -/+ & $150 / 40$ \\
\hline Never smoker & $120(63.2)$ \\
\hline Ex-smoker & $39(20.5)$ \\
\hline Current smoker & $31(16.3)$ \\
\hline Usage of RAS inhibitors & $70(36.8)$ \\
\hline Energy intake/day, kcal & $1657.4(749.0)$ \\
\hline Carbohydrate intake, g/day & $200.8(90.1)$ \\
\hline Protein intake, g/day & $66.8(36.2)$ \\
\hline Total fat intake, g/day & $51.6(28.4)$ \\
\hline Saturated fatty acids intake, g/day & $13.1(8.2)$ \\
\hline Monosaturated fatty acids intake, g/day & $18.7(10.3)$ \\
\hline Polyunsaturated fatty acids intake, g/day & $12.7(5.3)$ \\
\hline n-3 fatty acids intake, g/day & $2.8(1.5)$ \\
\hline $\mathrm{n}-6$ fatty acids intake, $\mathrm{g} /$ day & $9.7(5.7)$ \\
\hline Total fiber intake, g/day & $11.5(7.0)$ \\
\hline Cholesterol intake, g/day & $388.0(269.0)$ \\
\hline Alcohol intake, g/day & $0.0(3.4)$ \\
\hline Sodium intake, g/day & $10.7(5.3)$ \\
\hline
\end{tabular}

Data are expressed as number (\%) of subjects or median (IQR) $B M I$ body mass index, GFR glomerular filtration rate, IQR interquartile range, $N D R$ no diabetic retinopathy, PDR proliferative diabetic retinopathy, PPDR preproliferative diabetic retinopathy, $S D R$ simple diabetic retinopathy 
Table 2 Circulating fatty acids of the study patients

\begin{tabular}{|c|c|c|c|c|}
\hline Fatty acids, $\mu \mathrm{g} / \mathrm{ml}$ & All $(n=190)$ & Normo-/ Micro-albuminuria $(n=164)$ & Macro-albuminuria $(n=26)$ & $p$-value \\
\hline Caproic acid (C6:0) & $0.5(2.3)$ & $0.5(2.4)$ & $0.6(1.9)$ & 0.808 \\
\hline Caprylic acid (C8:0) & $0.5(2.8)$ & $0.5(2.6)$ & $1.1(4.0)$ & 0.245 \\
\hline Capric acid (C10:0) & $1.0(2.9)$ & $0.9(2.7)$ & $1.4(4.1)$ & 0.383 \\
\hline Undecyl acid (C11:0) & $1.4(1.9)$ & $1.4(1.8)$ & $1.3(2.3)$ & 0.662 \\
\hline Lauric acid (C12:0) & $5.4(8.0)$ & $5.3(7.5)$ & $6.1(11.0)$ & 0.640 \\
\hline Tridecyl acid (C13:0) & $2.5(2.5)$ & $2.6(2.5)$ & $2.0(2.8)$ & 0.230 \\
\hline Myristic acid (C14:0) & $27.4(16.8)$ & $27.616 .9)$ & $25.8(16.4)$ & 0.603 \\
\hline Myristoleic acid (C14:1(9c)) & $4.9(5.5)$ & $5.1(5.6)$ & $3.7(4.5)$ & 0.226 \\
\hline Pentadecanoic acid (C15:0) & $6.0(2.7)$ & $6.2(2.6)$ & $4.8(2.9)$ & 0.011 \\
\hline Pentadecenoic acid (C15:1) & $6.0(2.7)$ & $2.3(28.5)$ & $0.0(0.0)$ & 0.691 \\
\hline Palmitic acid (C16:0) & $743.5(314.8)$ & $747.9(315.9)$ & $715.4(312.4)$ & 0.626 \\
\hline Palmitoleic acid (C16:1(9c)) & $47.6(37.2)$ & $46.3(34.3)$ & $55.2(51.9)$ & 0.259 \\
\hline Stearic acid (C18:0) & $212.7(84.4)$ & $216.2(86.1)$ & $190.3(70.2)$ & 0.147 \\
\hline Petroselinic acid (C18:1(6c)) & $821.7(438.0)$ & $815.8(435.6)$ & $858.6(460.4)$ & 0.645 \\
\hline Oleic acid (C18:1(9c)) & $1009.0(853.4)$ & $1052.2(886.2)$ & $736.7(543.4)$ & 0.040 \\
\hline Vaccenic acid (C18:1(11 t)) & $27.5(150.3)$ & $31.9(161.4)$ & $0.0(0.0)$ & 0.316 \\
\hline$(C 18: 1(11 \mathrm{c}))$ & $57.3(33.0)$ & $58.1(31.7)$ & $52.6(40.8)$ & 0.431 \\
\hline Linolelaidic acid (C18:2(9t12c)) & $80.9(257.0)$ & $86.5(268.8)$ & $45.9(164.0)$ & 0.455 \\
\hline Linoleic acid (C18:2(9c12c)) & $868.1(370.9)$ & $878.8(381.4)$ & $800.5(293.6)$ & 0.319 \\
\hline$C 18: 3(9 t 12 t 15 c)+C 18: 3(9 t 12 c 15 t)$ & $7.1(9.4)$ & $7.6(9.6)$ & $3.9(7.5)$ & 0.032 \\
\hline ૪- Linolenic acid (C18:3n6) & $5.2(4.7)$ & $5.5(4.6)$ & $3.5(5.2)$ & 0.021 \\
\hline$C 18: 3(9 \mathrm{c} 12 \mathrm{t} 15 \mathrm{t})+\mathrm{C} 18: 3(9 \mathrm{c} 12 \mathrm{c} 15 \mathrm{t})$ & $9.5(7.4)$ & $10.0(7.2)$ & $6.3(7.9)$ & 0.009 \\
\hline $\mathrm{C} 18: 3(9 \mathrm{c} 12 \mathrm{t} 15 \mathrm{c})$ & $0.9(2.4)$ & $0.8(2.3)$ & $1.5(2.6)$ & 0.149 \\
\hline C18:3(9t12c15c) & $1.9(7.0)$ & $2.1(7.5)$ & $0.5(2.4)$ & 0.276 \\
\hline a-Linolenic acid (C18:3(9c12c15c)) & $22.2(10.4)$ & $22.8(10.5)$ & $18.8(8.7)$ & 0.034 \\
\hline Arachidic acid (C20:0) & $0.4(2.2)$ & $0.5(2.4)$ & $0.0(0.0)$ & 0.330 \\
\hline Eicosenoic acid (C20:1) & $6.7(3.8)$ & $7.1(3.5)$ & $4.3(4.3)$ & $<0.001$ \\
\hline Eicosadienoic acid (C20:2) & $9.5(4.3)$ & $9.9(4.2)$ & $7.5(4.1)$ & 0.007 \\
\hline Eicosatrienoic acid (C20:3 $(11,14,17))$ & $0.1(0.9)$ & $0.1(0.9)$ & $0.0(0.0)$ & 0.692 \\
\hline Dihomo-gamma-linolenic acid (C20:3n6) & $27.6(10.7)$ & $28.0(10.3)$ & $24.9(12.9)$ & 0.176 \\
\hline Arachidonic acid (C20:4n6) & $157.6(69.6)$ & $164.8(66.0)$ & $112.3(75.3)$ & $<0.001$ \\
\hline Eicosapentaenoic acid (C20:5n3) & $42.0(24.5)$ & $41.1(27.1)$ & $47.5(29.8)$ & 0.271 \\
\hline Heneicosylic acid (C21:0) & $4.5(4.7)$ & $5.1(4.7)$ & $1.2(3.1)$ & $<0.001$ \\
\hline Behenic acid (C22:0) & $13.2(8.6)$ & $14.0(8.3)$ & $8.7(9.2)$ & 0.004 \\
\hline Erucic acid (C22:1n9) & $1.9(4.5)$ & $2.0(4.6)$ & $1.3(3.6)$ & 0.432 \\
\hline Docosadienoic acid (C22:2) & $5.9(5.9)$ & $6.1(6.0)$ & $5.1(4.7)$ & 0.455 \\
\hline Docosahexaenoic acid (C22:6n3) & $146.1(63.9)$ & $147.1(64.1)$ & $140.0(63.7)$ & 0.593 \\
\hline Tricosanoic acid (C23:0) & $16.1(7.2)$ & $16.8(6.8)$ & $11.5(7.7)$ & $<0.001$ \\
\hline Lignoceric acid (C24:0) & $13.0(13.3)$ & $13.7(13.4)$ & $8.6(11.9)$ & 0.033 \\
\hline Nervonic acid (C24:1) & $3.4(6.0)$ & $3.7(6.2)$ & $1.9(3.6)$ & 0.159 \\
\hline
\end{tabular}

Data are expressed as mean (SD)

confidence interval (CI) of AA and other variables in the presence of macroalbuminuria were calculated by performing univariate and multivariate logistic regression analyses. We adjusted for age and sex (Model 2) and further adjusted for body mass index (BMI), disease duration, index of insulin resistance, total cholesterol, 
Table 3 Dietary fatty acids intake of the study patients

\begin{tabular}{|c|c|c|c|c|}
\hline & All $(n=190)$ & Normo-/ Micro-albuminuria $(n=164)$ & Macro-albuminuria $(n=26)$ & $p$-value \\
\hline Total energy intake, kcal/day & $1682.5(587.3)$ & $1649.6(551.3)$ & $1891.5(809.9)$ & 0.069 \\
\hline Carbohydrate intake, g/day & $209.9(80.6)$ & $204.8(70.8)$ & $233.3(129.2)$ & 0.117 \\
\hline Protein intake, g/day & $71.7(28.6)$ & $70.5(27.4)$ & $81.6(37.4)$ & 0.090 \\
\hline Total fat, g/day & $53.5(20.0)$ & $53.0(20.0)$ & $57.9(22.0)$ & 0.277 \\
\hline Total fiber intake, g/day & $12.0(5.1)$ & $12.0(4.9)$ & $12.7(7.0)$ & 0.546 \\
\hline Saturated fatty acid intake, g/day & $13.7(5.7)$ & $13.6(5.8)$ & $14.2(5.4)$ & 0.666 \\
\hline Monosaturated fatty acids intake, g/day & $19.3(7.5)$ & $19.1(7.5)$ & $21.3(8.4)$ & 0.192 \\
\hline Polyunsaturated fatty acids intake, g/day & $13.2(4.9)$ & $13.1(4.9)$ & $14.4(5.8)$ & 0.273 \\
\hline Caproic acid (C6:0) & $92.3(83.2)$ & $95.0(85.4)$ & $75.0(66.0)$ & 0.284 \\
\hline Caprylic acid (C8:0) & $90.8(85.6)$ & $94.7(89.4)$ & $65.1(48.7)$ & 0.123 \\
\hline Capric acid (C10:0) & $151.5(128.2)$ & $156.6(132.4)$ & $118.0(92.0)$ & 0.179 \\
\hline Lauric acid (C12:0) & $360.9(358.9)$ & $377.3(376.6)$ & $253.2(178.9)$ & 0.123 \\
\hline Myristic acid (C14:0) & $1040.4(563.6)$ & $1039.6(572.2)$ & $1045.4(512.8)$ & 0.963 \\
\hline Myristoleic acid (C14:1(9c)) & $77.8(43.6)$ & $77.2(44.2)$ & $81.9(39.9)$ & 0.627 \\
\hline Pentadecanoic acid (C15:0) & $99.4(52.4)$ & $98.6(52.7)$ & $104.7(50.9)$ & 0.605 \\
\hline Palmitic acid (C16:0) & $8234.1(3281.5)$ & $8159.4(3291.8)$ & $8724.8(3241.2)$ & 0.443 \\
\hline Palmitoleic acid (C16:1(9c)) & $873.4(394.2)$ & $847.8(382.3)$ & $1041.7(437.5)$ & 0.028 \\
\hline Stearic acid (C18:0) & $3007.0(1250.8)$ & $2981.8(1255.6)$ & $3172.2(1233.5)$ & 0.498 \\
\hline Oleic acid (C18:1(9c)) & $17,282.8(6881.2)$ & $17,066.6(6782.7)$ & $18,701.6(7503.6)$ & 0.290 \\
\hline Linoleic acid (C18:2(9c12c)) & $9975.4(3823.8)$ & $9898.3(3745.0)$ & $10,481.8(4364.0)$ & 0.497 \\
\hline$\gamma$ - Linolenic acid (C18:3n6) & $7.5(5.1)$ & $7.4(5.0)$ & $8.1(5.8)$ & 0.573 \\
\hline a-Linolenic acid (C18:3(9c12c15c)) & $1634.0(684.6)$ & $1616.7(664.1)$ & $1747.6(814.3)$ & 0.395 \\
\hline Arachidic acid (C20:0) & $154.1(60.3)$ & $152.5(58.9)$ & $165.1(69.5)$ & 0.350 \\
\hline Eicosenoic acid (C20:1) & $564.5(302.7)$ & $540.9(279.5)$ & $718.8(398.8)$ & 0.008 \\
\hline Eicosadienoic acid (C20:2) & $49.3(21.9)$ & $48.2(21.6)$ & $56.1(23.2)$ & 0.107 \\
\hline Dihomo-gamma-linolenic acid (C20:3n6) & $31.9(14.0)$ & $31.6(13.8)$ & $37.0(14.6)$ & 0.063 \\
\hline Arachidonic acid (C20:4n6) & $188.9(82.1)$ & $183.6(80.3)$ & $223.3(87.8)$ & 0.031 \\
\hline Eicosapentaenoic acid (C20:5n3) & $402.9(277.5)$ & $385.0(267.2)$ & $520.1(319.9)$ & 0.029 \\
\hline Behenic acid (C22:0) & $79.7(32.6)$ & $79.0(31.7)$ & $83.8(38.4)$ & 0.512 \\
\hline Erucic acid (C22:1n9) & $406.3(309.9)$ & $383.7(24.9)$ & $548.6(62.4)$ & 0.015 \\
\hline Docosahexaenoic acid (C22:6n3) & $664.6(422.8)$ & $636.6(404.4)$ & $848.9(499.7)$ & 0.025 \\
\hline Lignoceric acid (C24:0) & $32.6(14.1)$ & $32.1(13.8)$ & $35.5(16.4)$ & 0.280 \\
\hline Nervonic acid (C24:1) & $59.8(36.4)$ & $57.1(34.4)$ & $77.9(44.2)$ & 0.010 \\
\hline
\end{tabular}

Data are expressed as mean (SD)

systolic blood pressure, smoking status, physical activity, total energy, usage of renin-angiotensin system inhibitors, creatinine and the history of CVD (Model 3). In addition, the area under the curve (AUC) of circulating arachidonic acid concentration for the presence of macroalbuminuria was calculated by the receiver operating characteristic (ROC) curve.

\section{Results}

In this study, the BDHQ was administered to 426 patients (234 men and 192 women) with T2DM. Among them, we measured circulating FAs in 197 patients (96 men and 101 women) using the GC/MS system. Moreover, we excluded 7 patients with EPA medication usage (Fig. 1).

The clinical characteristics of the 190 patients with T2DM are summarized in Table 1. Mean age, HbA1c, and UAE were $62.7 \pm 12.1$ years, $7.5 \pm 1.6 \%$, and $202.0 \pm$ $583.0 \mathrm{mg} / \mathrm{gCr}$, respectively. The number (percentage) of patients with macroalbuminuria was 27 (13.7\%).

Mean circulating FA concentrations are shown in Table 2. Pentadecanoic acid (C15:0), oleic acid (C18: 
Table 4 Correlation coefficient between logarithm urinary albumin excretion and serum fatty acids

\begin{tabular}{|c|c|c|}
\hline Fatty acids, $\mu \mathrm{g} / \mathrm{ml}$ & $r$ & $p$-value \\
\hline Caproic acid (C6:0) & 0.042 & 0.563 \\
\hline Caprylic acid (C8:0) & 0.114 & 0.118 \\
\hline Capric acid (C10:0) & -0.044 & 0.546 \\
\hline Undecyl acid (C11:0) & -0.018 & 0.802 \\
\hline Lauric acid (C12:0) & 0.043 & 0.553 \\
\hline Tridecyl acid (C13:0) & -0.026 & 0.727 \\
\hline Myristic acid (C14:0) & 0.011 & 0.882 \\
\hline Myristoleic acid (C14:1(9c)) & 0.105 & 0.148 \\
\hline Pentadecanoic acid (C15:0) & -0.200 & 0.007 \\
\hline Pentadecenoic acid (C15:1) & -0.030 & 0.675 \\
\hline Palmitic acid (C16:0) & -0.010 & 0.850 \\
\hline Palmitoleic acid (C16:1(9c)) & 0.029 & 0.688 \\
\hline Stearic acid (C18:0) & -0.070 & 0.308 \\
\hline Petroselinic acid (C18:1(6c)) & 0.022 & 0.766 \\
\hline Oleic acid (C18:1(9c)) & -0.081 & 0.268 \\
\hline Vaccenic acid (C18:1(11 t)) & -0.051 & 0.487 \\
\hline$(C 18: 1(11 \mathrm{c}))$ & -0.061 & 0.406 \\
\hline Linolelaidic acid (C18:2(9t12c)) & -0.038 & 0.606 \\
\hline Linoleic acid (C18:2(9c12c)) & -0.072 & 0.321 \\
\hline$C 18: 3(9 t 12 t 15 c)+C 18: 3(9 t 12 c 15 t)$ & -0.129 & 0.075 \\
\hline$\gamma$ - Linolenic acid (C18:3n6) & -0.089 & 0.220 \\
\hline$C 18: 3(9 c 12 t 15 t)+C 18: 3(9 c 12 c 15 t)$ & -0.132 & 0.069 \\
\hline $\mathrm{C} 18: 3(9 \mathrm{c} 12 \mathrm{t} 15 \mathrm{c})$ & 0.100 & 0.169 \\
\hline $\mathrm{C} 18: 3(9 \mathrm{t} 12 \mathrm{c} 15 \mathrm{c})$ & -0.029 & 0.688 \\
\hline a-Linolenic acid (C18:3(9c12c15c)) & -0.135 & 0.064 \\
\hline Arachidic acid (C20:0) & -0.061 & 0.402 \\
\hline Eicosenoic acid (C20:1) & -0.002 & 0.977 \\
\hline Eicosadienoic acid (C20:2) & -0.175 & 0.016 \\
\hline Eicosatrienoic acid (C20:3 $(11,14,17))$ & 0.084 & 0.249 \\
\hline Dihomo-gamma-linolenic acid (C20:3n6) & -0.100 & 0.171 \\
\hline Arachidonic acid (C20:4n6) & -0.221 & 0.002 \\
\hline Eicosapentaenoic acid (C20:5n3) & 0.065 & 0.376 \\
\hline Heneicosylic acid (C21:0) & 0.021 & 0.776 \\
\hline Behenic acid (C22:0) & -0.183 & 0.011 \\
\hline Erucic acid (C22:1n9) & -0.014 & 0.847 \\
\hline Docosadienoic acid (C22:2) & -0.020 & 0.787 \\
\hline Docosahexaenoic acid (C22:6n3) & -0.027 & 0.709 \\
\hline Tricosanoic acid (C23:0) & -0.195 & 0.007 \\
\hline Lignoceric acid (C24:0) & -0.149 & 0.040 \\
\hline Nervonic acid (C24:1) & -0.062 & 0.396 \\
\hline
\end{tabular}

1(9c)), C18:3(9c12t15t) + C18:3(9c12c15t), $\alpha$-Linolenic acid (C18:3(9c12c15c)), eicosenoic acid (C20:1), eicosadienoic acid (C20:2), AA (C20:4n6), heneicosylic acid (C21:0), behenic acid (C22:0), and tricosanoic acid (C23: 0 ) were significantly lower in patients with macroalbuminuria than in those without macroalbuminuria.

In addition, the mean dietary intakes of nutrients, including FAs, are shown in Table 3. Energy intake in patients with macroalbuminuria tended to be higher than that in patients without macroalbuminuria (1891.5 \pm $809.9 \mathrm{kcal} /$ day vs. $1649.6 \pm 551.3 \mathrm{kcal} /$ day, $\quad p=0.069)$, whereas total fat intake was not significantly different $(57.9 \pm 22.0 \mathrm{~g} /$ day vs. $53.0 \pm 20.0 \mathrm{~g} /$ day, $p=0.277)$. The dietary intake of eicosenoic acid (C20:1), AA (C20:4n6), EPA (C20:5n3), erucic acid (C22:1n9), erucic acid (C22: 1n9), docosahexaenoic acid (C22:6n3), and nervonic acid (C24:1) in patients with macroalbuminuria was significantly higher than that in patients without macroalbuminuria.

Moreover, we investigated the association between $\log$ circulating FAs and log UAE. Logarithm AA showed a significant correlation $(r=-0.221, p=0.002)$ (Table 4). Therefore, we performed logistic regression analyses to investigate the association between logarithm AA and the presence of macroalbuminuria. In univariate logistic regression analysis, logarithm AA was negatively associated with the presence of macroalbuminuria (Model 1, OR of $\Delta 1$ incremental: $0.03,95 \%$ CI: $0.01-0.21$, $p<0.001)$. Multivariate logistic regression analysis revealed that logarithm AA was negatively associated with the presence of macroalbuminuria, even after adjusting for covariates (OR of $\Delta 1$ incremental: $0.32,95 \% \mathrm{CI}$ : 0.10-0.99, $p=0.042$ ) (Table 5).

Additionally, in ROC analyses, AUC of circulating arachidonic acid concentration was 0.714 (95\% CI 0.6010.827 ) and the cut-off level of serum AAs with diabetic patients for predicting the presence of macroalbuminuria was $327.5 \mu \mathrm{g} / \mathrm{ml}$ (Fig. 2).

\section{Discussion}

In the present study, in a cohort of 190 Japanese individuals with T2DM, we investigated the association between circulating FAs and diabetic nephropathy, and we revealed that circulating AA is negatively associated with UAE and the presence of macroalbuminuria.

Possible explanations for the association between circulating AA and diabetic nephropathy are described below. AA is a component of biological membranes and is involved in the generation of prostaglandins, which are the most representative physiologically active lipids, through cyclooxygenase (COX). Prostacyclin (PGI2), a type of prostaglandin, has vasodilatory effects [27], antiplatelet effects [28], angiogenesis [29], and protective effects on vascular endothelium [30]. PGI2 is produced 
Table 5 Logistic regression analyses for macroalbuminuria

\begin{tabular}{|c|c|c|c|c|c|c|}
\hline & \multicolumn{2}{|l|}{ Model 1} & \multicolumn{2}{|l|}{ Model 2} & \multicolumn{2}{|l|}{ Model 3} \\
\hline & Odds ratio $(95 \% \mathrm{Cl})$ & $p$-value & Odds ratio $(95 \% \mathrm{Cl})$ & $p$-value & Odds ratio $(95 \% \mathrm{Cl})$ & $p$-value \\
\hline Age, yrs & $1.07(1.03-1.13)$ & $<0.001$ & $1.06(1.01-1.12)$ & 0.015 & $1.03(0.94-1.13)$ & 0.509 \\
\hline Men & $4.43(1.70-11.5)$ & $<0.001$ & $3.38(1.23-9.32)$ & 0.019 & $4.73(0.47-47.5)$ & 0.186 \\
\hline Disease duration, yrs & $1.06(1.01-1.10)$ & 0.010 & - & - & $1.08(0.96-1.22)$ & 0.180 \\
\hline Body mass index, $\mathrm{kg} / \mathrm{m}^{2}$ & $0.99(0.91-1.09)$ & 0.878 & - & - & $1.05(0.83-1.32)$ & 0.684 \\
\hline 20/(fasting C-peptide $\times$ fasting plasma glucose) & $0.99(0.93-1.06)$ & 0.596 & - & - & $1.03(0.82-1.29)$ & 0.790 \\
\hline Creatinine, $\mu \mathrm{mol} / \mathrm{L}$ & $1.07(1.04-1.09)$ & $<0.001$ & - & - & $1.07(1.02-1.15)$ & $<0.001$ \\
\hline Total cholesterol, mmol/L & $0.81(0.54-1.21)$ & 0.311 & & & $1.05(0.40-2.74)$ & 0.921 \\
\hline Systolic blood pressure, $\mathrm{mmHg}$ & $1.03(1.01-1.05)$ & 0.003 & - & - & $1.02(0.96-1.08)$ & 0.590 \\
\hline Never smoker & 1.00 (Reference) & - & - & - & 1.00 (Reference) & - \\
\hline Ex-smoker & $2.90(1.02-8.22)$ & 0.045 & - & - & $0.96(0.11-8.13)$ & 0.974 \\
\hline Current smoker & $3.01(1.14-7.91)$ & 0.026 & - & - & $2.54(0.31-20.6)$ & 0.381 \\
\hline Exercise & $0.76(0.33-1.76)$ & 0.527 & - & - & $0.38(0.07-1.96)$ & 0.248 \\
\hline Total Energy, kcal/day & $1.00(0.99-1.00)$ & 0.095 & - & - & $0.99(0.99-1.00)$ & 0.431 \\
\hline RAS inhibitors & $4.85(1.98-11.87)$ & $<0.001$ & - & - & $5.04(0.92-27.6)$ & 0.062 \\
\hline History of CVD & $7.57(2.02-28.4)$ & 0.004 & & & $1.52(0.02-99.7)$ & 0.844 \\
\hline Log arachidonic acid & $0.03(0.01-0.21)$ & $<0.001$ & $0.46(0.24-0.87)$ & 0.016 & $0.32(0.10-0.99)$ & 0.042 \\
\hline
\end{tabular}

Model 1 was univariate; Model 2: adjusted for age, sex; Model 3: model 2 plus body mass index, disease duration, 20/(fasting C-peptide $\times$ fasting plasma glucose), uric acids, total cholesterol, systolic blood pressure, smoking status, physical activity, total energy, RAS inhibitors usage, and history of CVD CVD cardiovascular disease, RAS renin-angiotensin system, UAE urinary albumin excretion

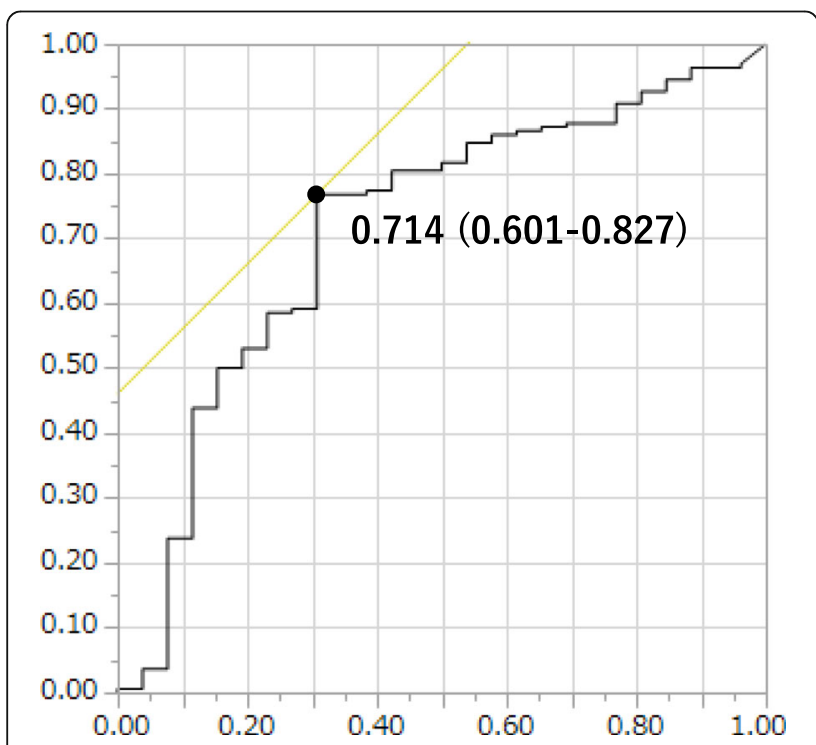

Fig. 2 Area under the receiver operating characteristic (ROC) curve (AUC) [95\% confidence interval (CI)] of several factors for the presence of macroalbuminuria. AUC of circulating arachidonic acid concentration was 0.714 (95\% Cl 0.601-0.827), and the cut-off level of serum AAs with diabetic patients for predicting the presence of macroalbuminuria was $327.5 \mu \mathrm{g} / \mathrm{ml}$ from prostaglandin $\mathrm{H} 2$ (PGH2) by prostacyclin synthase [31]. In addition, prostaglandin E1 has a protective effect on diabetic kidney disease by decreasing UAE [32], whereas prostaglandin E2 decrease myocardium contractility and cause cardiorenal syndrome [33]. AA is associated with $\mathrm{PGH} 2$, through separation from phospholipids in the cell membrane and receiving COX. PGI2 is well known for its regulation of renal hemodynamics, tubular transport, and renin release and plays an important role by coupling with its receptors and the downstream signals in various types of renal diseases including chronic kidney disease [34]. Therefore, circulating AA levels in patients with macroalbuminuria were significantly lower than those with normoalbuminuria and microalbuminuria, suggesting that the body promoted the production of PGI2 to prevent progression of nephropathy and the other CVD.

On the contrary, AA has been reported to potentiate hypoxia-induced vascular endothelial growth factor (VEGF) expression through the Notch-1, Wnt-1, and HIF-1alpha pathways [35]. In several previous studies, VEGF was proven to have an ameliorating effect on diabetic nephropathy [36], normalization of hyperpermeability in the glomeruli of diabetic nephropathy [37], and in restoring endothelial glycocalyx in diabetic nephropathy [38]. It is suggested that AA is consumed for PGI2 production and the concentration of AA in the body is lowered, which may reduce the effects of VEGF from being fully activated. Collectively, patients with advanced 
diabetic nephropathy might consume more AA to raise levels of PGI2, which leads to lower circulating AA levels and decreased AA reduce the effect of VEGF and induces further progression of nephropathy. This is potentially the reason why circulating AA levels in patients with diabetic nephropathy are significantly lower than those of patients without this condition, although dietary AA intake in patients with diabetic nephropathy is higher than that in patients without diabetic nephropathy.

There are three major limitations in this study that could be addressed in future research. First, this study had a cross-sectional design; thus, further studies are required to establish the causal relationship between circulating AA levels and diabetic nephropathy. Second, the sample size in this study was relatively small. Thus, further large-scale studies are needed. Third, we did not check circulating prostaglandin level.

\section{Conclusions}

In conclusion, this study provides the first demonstration that circulating AA levels are negatively associated with UAE and that these levels are significantly lower in patients with macroalbuminuria than in those without macroalbuminuria. Future prospective studies on the relationship between circulating AA levels and the incidence of diabetic nephropathy and the differences in mechanism between dietary AA and circulating AA are required.

\section{Supplementary Information}

The online version contains supplementary material available at https://doi. org/10.1186/s12882-021-02271-8.

\section{Additional file 1:.}

Additional file 2:.

\section{Abbreviations}

AA: Arachidonic acid; BDHQ: Brief-type self-administered diet history questionnaire; BMI: Body mass index; Cl: Confidence interval; COX: Cyclooxygenase; EPA: Eicosapentaenoic acid; FA: Fatty acid; HbA1c: Hemoglobin A1c; PGH2: Prostaglandin H2; PGI2: Prostacyclin; PUFA: Polyunsaturated fatty acid; T2DM: Type 2 diabetes mellitus; UAE: Urinary albumin excretion; VEGF: Vascular endothelial growth factor

\section{Acknowledgments}

We thank all of the staff members in Kyoto Prefectural University of Medicine. We would like to thank Editage (www.editage.com) for English language editing.

\footnotetext{
Authors' contributions

T.O. originated and designed the study, researched the data, and wrote the manuscript. Y. H. originated and designed the study, researched the data, and reviewed the manuscript. H.N., S.M., T.S., E.U., N.N., M.H., M.A., and M.Y. researched the data and contributed to the discussion. M.F. originated and designed the study researched the data and reviewed and edited the manuscript. Y.H. is the guarantor of this work and, as such, had full access to all the data in the study and takes responsibility for the integrity of the data and the accuracy of the data analysis. All authors were involved in the writing of the manuscript and approved the final version of this article. The authors read and approved the final manuscript.
}

Funding

None.

\section{Availability of data and materials}

The datasets used and/or analysed during the current study are available from the corresponding author on reasonable request.

\section{Ethics approval and consent to participate}

This study was approved by the Ethics Committee of Kyoto Prefectural University of Medicine (Kyoto, Japan) and Kameoka Municipal Hospital (Kameoka, Japan) (IRB number: RBMR-E-466). The informed consent obtained from study participants was written.

\section{Consent for publication}

Not applicable.

\section{Competing interests}

Yoshitaka Hashimoto has received grants from Asahi Kasei Pharma, personal fees from Daiichi Sankyo Co., Ltd., personal fees from Mitsubishi Tanabe Pharma Corp., personal fees from Sanofi K.K., personal fees from Novo Nordisk Pharma Ltd., outside the submitted work. Takafumi Senmaru has received personal fees from Ono Pharma Co., Ltd., Mitsubishi Tanabe Pharma Co, Astellas Pharma Inc., Kyowa Hakko Kirin Co., Ltd., Sanofi K.K., MSD K.K., Kowa Pharma Co., Ltd., Taisho Toyama Pharma Co., Ltd., Takeda Pharma Co., Ltd., Kissei Pharma Co., Ltd., Novo Nordisk Pharma Ltd., Eli Lilly Japan K.K. outside the submitted work. Emi Ushigome has received grants from the Japanese Study Group for Physiology and Management of Blood Pressure, the Astellas Foundation for Research on Metabolic Disorders (Grant number: 4024). Donated Fund Laboratory of Diabetes therapeutics is an endowment department, supported with an unrestricted grant from Ono Pharmaceutical Co., Ltd., and received personal fees from AstraZeneca plc, Astellas Pharma Inc., Daiichi Sankyo Co., Ltd., Kyowa Hakko Kirin Company Ltd., Kowa Pharmaceutical Co., Ltd., MSD K.K., Mitsubishi Tanabe Pharma Corp., Novo Nordisk Pharma Ltd., Taisho Toyama Pharmaceutical Co., Ltd., Takeda Pharmaceutical Co., Ltd., Nippon Boehringer Ingelheim Co., Ltd., and Sumitomo Dainippon Pharma Co., Ltd., outside the submitted work. Masahide Hamaguchi has received grants from Asahi Kasei Pharma, Nippon Boehringer Ingelheim Co., Ltd., Mitsubishi Tanabe Pharma Corporation, Daiichi Sankyo Co., Ltd., Sanofi K.K., Takeda Pharmaceutical Company Limited, Astellas Pharma Inc., Kyowa Kirin Co., Ltd., Sumitomo Dainippon Pharma Co., Ltd., Novo Nordisk Pharma Ltd., and Eli Lilly Japan K.K., outside the submitted work. Mai Asano received personal fees from Novo Nordisk Pharma Ltd., Abbott Japan Co., Ltd., AstraZeneca plc, Kowa Pharmaceutical Co., Ltd., Ono Pharmaceutical Co., Ltd., Takeda Pharmaceutical Co., Ltd., outside the submitted work. Masahiro Yamazaki reports personal fees from MSD K.K., Sumitomo Dainippon Pharma Co., Ltd., Kowa Company, Limited, AstraZeneca PLC, Takeda Pharmaceutical Company Limited, Kyowa Hakko Kirin Co., Ltd., Daiichi Sankyo Co., Ltd., Kowa Pharmaceutical Co., Ltd., Ono Pharma Co., Ltd., outside the submitted work. Michiaki Fukui has received grants from Nippon Boehringer Ingelheim Co., Ltd., Kissei Pharma Co., Ltd., Mitsubishi Tanabe Pharma Co, Daiichi Sankyo Co., Ltd., Sanofi K.K., Takeda Pharma Co., Ltd., Astellas Pharma Inc., MSD K.K., Kyowa Hakko Kirin Co., Ltd., Sumitomo Dainippon Pharma Co., Ltd., Kowa Pharmaceutical Co., Ltd., Novo Nordisk Pharma Ltd., Ono Pharma Co., Ltd., Sanwa Kagaku Kenkyusho Co., Ltd. Eli Lilly Japan K.K., Taisho Pharma Co., Ltd., Terumo Co., Teijin Pharma Ltd., Nippon Chemiphar Co., Ltd., and Johnson \& Johnson K.K. Medical Co., Abbott Japan Co., Ltd., and received personal fees from Nippon Boehringer Ingelheim Co., Ltd., Kissei Pharma Co., Ltd., Mitsubishi Tanabe Pharma Corp., Daiichi Sankyo Co., Ltd., Sanofi K.K., Takeda Pharma Co., Ltd., Astellas Pharma Inc., MSD K.K., Kyowa Kirin Co., Ltd., Sumitomo Dainippon Pharma Co., Ltd., Kowa Pharma Co., Ltd., Novo Nordisk Pharma Ltd., Ono Pharma Co., Ltd., Sanwa Kagaku Kenkyusho Co., Ltd., Eli Lilly Japan K.K., Taisho Pharma Co., Ltd., Bayer Yakuhin, Ltd., AstraZeneca K.K., Mochida Pharma Co., Ltd., Abbott Japan Co., Ltd., Medtronic Japan Co., Ltd., Arkley Inc., Teijin Pharma Ltd. and Nipro Cor., outside the submitted work

\section{Author details}

'Department of Endocrinology and Metabolism, Kyoto Prefectural University of Medicine, Kyoto 602-8566, Japan. ${ }^{2}$ Agilent Technologies, Chromatography Mass Spectrometry Sales Department, Life Science and Applied Markets Group, Tokyo 192-8510, Japan. 
Received: 15 June 2020 Accepted: 16 February 2021 Published online: 23 February 2021

\section{References}

1. Liyanage T, Ninomiya T, Jha V, Neal B, Patrice HM, Okpechi I, et al. Worldwide access to treatment for end-stage kidney disease: a systematic review. Lancet. 2015;385:1975-82 https://doi.org/10.1016/S0140-6736(14)61 $601-9$

2. Holman RR, Paul SK, Bethel MA, Neil HAW, Matthews DR. Long-term followup after tight control of blood pressure in type 2 diabetes. N Engl J Med. 2008;359:1565-76 https://doi.org/10.1056/NEJMoa0806359.

3. Afkarian M, Sachs MC, Kestenbaum B, Hirsch IB, Tuttle KR, Himmelfarb J, et al. Kidney disease and increased mortality risk in type 2 diabetes. J Am Soc Nephrol. 2013;24:302-8 https://doi.org/10.1681/ASN.2012070718.

4. Thomas ME, Schreiner GF. Contribution of proteinuria to progressive renal injury: consequences of tubular uptake of fatty acid bearing albumin. Am J Nephrol. 1993;13:385-98 https://doi.org/10.1159/000168653.

5. Soumura M, Kume S, Isshiki K, Takeda N, Araki SI, Tanaka Y, et al. Oleate and eicosapentaenoic acid attenuate palmitate-induced inflammation and apoptosis in renal proximal tubular cell. Biochem Biophys Res Commun. 2010;402:265-71 https://doi.org/10.1016/j.bbrc.2010.10.012.

6. Cobbs A, Chen X, Zhang Y, George J, Huang M bo, Bond V, et al. Saturated fatty acid stimulates production of extracellular vesicles by renal tubular epithelial cells. Mol Cell Biochem. 2019;458:113-24 https://doi.org/10.1007/ s11010-019-03535-6.

7. Dyerberg J, Bang HO, Stoffersen E, Moncada S, Vane JR. Eicosapentaenoic acid and prevention of thrombosis and atherosclerosis? Lancet. 1978;312: 117-9 https://doi.org/10.1016/S0140-6736(78)91505-2.

8. Yokoyama M, Origasa H, Matsuzaki M, Matsuzawa Y, Saito Y, Ishikawa Y, et al. Effects of eicosapentaenoic acid on major coronary events in hypercholesterolaemic patients (JELIS): a randomised open-label, blinded endpoint analysis. Lancet. 2007;369:1090-8 https://doi.org/10.1016/S01406736(07)60527-3.

9. Rissanen T, Voutilainen S, Nyyssonen K, Lakka TA, Salonen JT. Fish oilderived fatty acids, docosahexaenoic acid and docosapentaenoic acid, and the risk of acute coronary events: the Kuopio Ischaemic heart disease risk factor study. Circulation. 2000;102:2677-9 https://doi.org/10.1161/01.CIR.1 02.22.2677.

10. Brash AR. Arachidonic acid as a bioactive molecule. J Clin Invest. 2001;107: 1339-45 https://doi.org/10.1172/JCl13210.

11. Ninomiya $T$, Nagata $M$, Hata J, Hirakawa $Y$, Ozawa M, Yoshida D, et al. Association between ratio of serum eicosapentaenoic acid to arachidonic acid and risk of cardiovascular disease: the Hisayama study. Atherosclerosis. 2013;231:261-7 https://doi.org/10.1016/j.atherosclerosis.2013.09.023.

12. Yagi S, Aihara Kl, Fukuda D, Takashima A, Bando M, Hara T, et al. Reduced ratio of eicosapentaenoic acid and docosahexaenoic acid to arachidonic acid is associated with early onset of acute coronary syndrome. Nutr J 2015;14 https://doi.org/10.1186/s12937-015-0102-4.

13. Domei T, Yokoi H, Kuramitsu S, Soga Y, Arita T, Ando K, et al. Ratio of serum n-3 to n-6 polyunsaturated fatty acids and the incidence of major adverse cardiac events in patients undergoing percutaneous coronary intervention. Circ J. 2012;76:423-9 https://doi.org/10.1253/circj.CJ-11-0941.

14. Harris WS, Mozaffarian D, Rimm E, Kris-Etherton P, Rudel LL, Appel LJ, et al. Omega-6 fatty acids and risk for cardiovascular disease: a science advisory from the American Heart Association Nutrition Subcommittee of the Council on Nutrition, Physical Activity, and Metabolism; Council on Cardiovascular Nursing; and Council on Epidemiology and Prevention. Circulation. 2009;119:902-7. https://doi.org/10.1161/CIRCULATIONAHA.108.1 91627.

15. Wu JHY, Lemaitre RN, King IB, Song $X$, Psaty BM, Siscovick DS, et al. Circulating omega- 6 polyunsaturated fatty acids and total and causespecific mortality: the cardiovascular health study. Circulation. 2014;130: 1245-53 https://doi.org/10.1161/CIRCULATIONAHA.114.011590.

16. Li M, Xu C, Shi J, Ding J, Wan X, Chen D, et al. Fatty acids promote fatty liver disease via the dysregulation of 3-mercaptopyruvate sulfurtransferase/ hydrogen sulfide pathway. Gut. 2018;67:2169-80 https://doi.org/10.1136/ gutjnl-2017-313778.

17. Tao M, McDowell MA, Saydah SH, Eberhardt MS. Relationship of polyunsaturated fatty acid intake to peripheral neuropathy among adults with diabetes in the National Health and nutrition examination survey
(NHANES) 1999-2004. Diabetes Care. 2008;31:93-5 https://doi.org/10.2337/ dc07-0931.

18. Sacks FM, Lichtenstein AH, Wu JHY, Appel LJ, Creager MA, Kris-Etherton PM, et al. Dietary fats and cardiovascular disease: a presidential advisory from the American Heart Association. Circulation. 2017;136:e1-23 https://doi. org/10.1161/CIR.0000000000000510.

19. Sakai R, Hashimoto $Y$, Ushigome E, Miki A, Okamura T, Matsugasumi M, et al. Late-night-dinner is associated with poor glycemic control in people with type 2 diabetes: the KAMOGAWA-DM cohort study. Endocr J. 2017; https:// doi.org/10.1507/endocrj.EJ17-0414.

20. Expert Committee on the Diagnosis and Classification of Diabetes Mellitus. Report of the expert committee on the diagnosis and classification of diabetes mellitus. Diab Care. 2003;26:S5-20. https://doi.org/10.2337/diacare.2 6.2007.s5

21. Ohkura T, Shiochi H, Fujioka Y, Sumi K, Yamamoto N, Matsuzawa K, et al. 20/ (fasting (-peptide $\times$ fasting plasma glucose) is a simple and effective index of insulin resistance in patients with type 2 diabetes mellitus: a preliminary report. Cardiovasc Diabetol. 2013;12:21. https://doi.org/10.1186/1475-284 0-12-21.

22. Wada T, Haneda M, Furuichi K, Babazono T, Yokoyama H, Iseki K, et al. Clinical impact of albuminuria and glomerular filtration rate on renal and cardiovascular events, and all-cause mortality in Japanese patients with type 2 diabetes. Clin Exp Nephrol. 2014;18:613-20 https://doi.org/10.1007/s10157013-0879-4.

23. Tesfaye S, Boulton AJM, Dyck PJ, Freeman R, Horowitz M, Kempler P, et al. Diabetic neuropathies: update on definitions, diagnostic criteria, estimation of severity, and treatments. Diabetes Care. 2010;33:2285-93 https://doi.org/1 $0.2337 / \mathrm{dc} 10-1303$

24. Kobayashi S, Murakami K, Sasaki S, Okubo H, Hirota N, Notsu A, et al. Comparison of relative validity of food group intakes estimated by comprehensive and brief-type self-administered diet history questionnaires against 16 d dietary records in Japanese adults. Public Health Nutr. 2011;14: 1200-11 https://doi.org/10.1017/\$1368980011000504.

25. Ministry of Education, Culture, Sports $S$ and T 2010 (in J. STANDARD TABLES OF FOOD COMPOSITION IN JAPAN - 2015 - (Seventh Revised Version). 2010.

26. Okamura T, Hamaguchi M, Bamba R, Nakajima H, Yoshimura Y, Kimura T, et al. Immune modulating effects of additional supplementation of estradiol combined with testosterone in murine testosterone-deficient NAFLD model Am J Physiol Liver Physiol 2020:ajpgi.00310.2019. https://doi.org/10.1152/a jpgi.00310.2019.

27. Manoogian C, Nadler J, Ehrlich L, Horton R. The renal vasodilating effect of dopamine is mediated by calcium flux and prostacyclin release in man. J Clin Endocrinol Metab. 1988;66:678-83 https://doi.org/10.1210/jcem-66-4-678.

28. Schrör K, Darius H, Matzky R, Ohlendorf R. The antiplatelet and cardiovascular actions of a new carbacyclin derivative (ZK 36 374) equipotent to PGI2 in vitro. Naunyn Schmiedeberg's Arch Pharmacol. 1981; 316:252-5 https://doi.org/10.1007/BF00505658.

29. Krishnamurthy P, Bird IM, Sheppard C, Magness RR. Effects of angiogenic growth factors on endothelium-derived prostacyclin production by ovine uterine and placental arteries. Prostaglandins Other Lipid Mediat. 1999;57:112 https://doi.org/10.1016/S0090-6980(98)00066-5.

30. Birukova AA, Zagranichnaya T, Fu P, Alekseeva E, Chen W, Jacobson JR, et al Prostaglandins PGE2 and PGI2 promote endothelial barrier enhancement via PKA- and Epac1/Rap1-dependent Rac activation. Exp Cell Res. 2007;313: 2504-20 https://doi.org/10.1016/j.yexcr.2007.03.036.

31. Lianos EA. Biosynthesis and role of arachidonic acid metabolites in glomerulonephritis. Nephron. 1984;37:73-7 https://doi.org/10.1159/0001 83217.

32. Wang H, Deng JL, Yue J, Li J, Hou YB. Prostaglandin E1 for preventing the progression of diabetic kidney disease. Cochrane Database Syst Rev. 2010;5: CD006872.

33. Gu X, Xu J, Zhu L, Bryson T, Yang XP, Peterson E, et al. Prostaglandin E2 Reduces Cardiac Contractility via EP3 Receptor. Circ Heart Fail. 2016;9: e003291. https://doi.org/10.1161/CIRCHEARTFAILURE.116.003291.

34. Li Y, Xia W, Zhao F, Wen Z, Zhang A, Huang S, et al. Prostaglandins in the pathogenesis of kidney diseases. Oncotarget. 2018;9:26586-602 https://doi. org/10.18632/oncotarget.25005.

35. Sang HL, Min HK, Ho JH. Arachidonic acid potentiates hypoxia-induced VEGF expression in mouse embryonic stem cells: involvement of notch, Wnt, and HIF-1a. Am J Physiol - Cell Physiol. 2009;297 https://doi.org/10.11 52/ajpcell.00579.2008. 
36. Falkevall A, Mehlem A, Palombo I, Heller Sahlgren B, Ebarasi L, He L, et al. Reducing VEGF-B signaling ameliorates renal lipotoxicity and protects against diabetic kidney disease. Cell Metab. 2017;25:713-26 https://doi.org/1 0.1016/j.cmet.2017.01.004.

37. Flyvbjerg A, Dagnæs-Hansen F, De Vriese AS, Schrijvers BF, Tilton RG, Rasch R. Amelioration of long-term renal changes in obese type 2 diabetic mice by a neutralizing vascular endothelial growth factor antibody. Diabetes. 2002;51:3090-4 https://doi.org/10.2337/diabetes.51.10.3090.

38. Oltean S, Qiu Y, Ferguson JK, Stevens M, Neal C, Russell A, et al. Vascular endothelial growth factor-A165b is protective and restores endothelial glycocalyx in diabetic nephropathy. J Am Soc Nephrol. 2015;26:1889-904 https://doi.org/10.1681/ASN.2014040350.

\section{Publisher's Note}

Springer Nature remains neutral with regard to jurisdictional claims in published maps and institutional affiliations.

Ready to submit your research? Choose BMC and benefit from:

- fast, convenient online submission

- thorough peer review by experienced researchers in your field

- rapid publication on acceptance

- support for research data, including large and complex data types

- gold Open Access which fosters wider collaboration and increased citations

- maximum visibility for your research: over $100 \mathrm{M}$ website views per year

At BMC, research is always in progress.

Learn more biomedcentral.com/submissions 